\title{
Geometrically nonlinear free and forced vibrations of Euler-Bernoulli multi-span beams
}

\author{
Hatim Fakhreddine ${ }^{1 *}$, Ahmed Adri ${ }^{1}$, Saïd Rifai $^{1}$ and Rhali Benamar ${ }^{2}$ \\ ${ }^{1}$ Laboratoire de Mécanique Productique et Génie Industriel, Ecole Supérieure de Technologie, \\ Hassan II University of Casablanca, B.P.8012, Oasis, Casablanca, Maroc \\ ${ }^{2}$ Mohammed V University in Rabat, EMI-Rabat, LERSIM, B.P.765 Agdal, Rabat, Morocco
}

\begin{abstract}
The objective of this paper is to establish the formulation of the problem of nonlinear transverse forced vibrations of uniform multi-span beams, with several intermediate simple supports and general end conditions, including use of translational and rotational springs at the ends. The beam bending vibration equation is first written at each span and then the continuity requirements at each simple support are stated, in addition to the beam end conditions. This leads to a homogeneous linear system whose determinant must vanish in order to allow nontrivial solutions to be obtained. The formulation is based on the application of Hamilton's principle and spectral analysis to the problem of nonlinear forced vibrations occurring at large displacement amplitudes, leading to the solution of a nonlinear algebraic system using numerical or analytical methods. The nonlinear algebraic system has been solved here in the case of a four span beam in the free regime using an approximate method developed previously (second formulation) leading to the amplitude dependent fundamental nonlinear mode of the multi-span beam and to the corresponding backbone curves. Considering the nonlinear regime, under a uniformly distributed excitation harmonic force, the calculation of the corresponding generalised forces has led to the conclusion that the nonlinear response involves predominately the fourth mode. Consequently, an analysis has been performed in the neighbourhood of this mode, based on the single mode approach, to obtain the multi-span beam nonlinear frequency response functions for various excitation levels.
\end{abstract}

\section{Introduction}

The analysis of structural vibration is of a crucial importance in many fields, such as aerospace, aeronautics, mechanical and civil engineering. Due to the improvement of the structure characteristics and the increasing use of new materials, the nonlinear vibration analysis is increasingly becoming a necessary step in the design and verification processes in many situations. The purpose of this work is to establish the formulation of the nonlinear transverse forced vibration of uniform multi-span beams with intermediate simple supports under various end conditions. The mathematical formulation of the problem has been derived using Hamilton's principle and spectral analysis to reduce the problem to solution of an

\footnotetext{
* Corresponding author: hatim.fakhreddine@gmail.com
} 
algebraic system, numerically or analytically. Different beam end conditions may be examined by the present formulation involving at the beam ends two types of springs i.e. Rotational and translational springs. The main aim of this study is to examine the effect of geometrical nonlinearity, occurring at large vibration amplitudes, as well as to extend the Benamar's method to the case of multi-span beams with intermediates simple supports. In addition, by the appropriate use of the single mode approach in the neighbourhood of the predominant mode excited by a uniformly distributed applied load, the nonlinear frequency response functions have been determined and plotted for various excitation levels.

\section{General formulation}

\subsection{Linear mode shapes of an Euler-Bernoulli multi-span beam:}

Consider a beam subjected to transverse vibrations and who's geometrical and mechanical characteristics, i.e. the length, width, thickness, second moment of area of cross-section, Young's modulus and the mass per unit length are respectively denoted by: $(L, \mathrm{~b}, h, I, E, \rho)$.

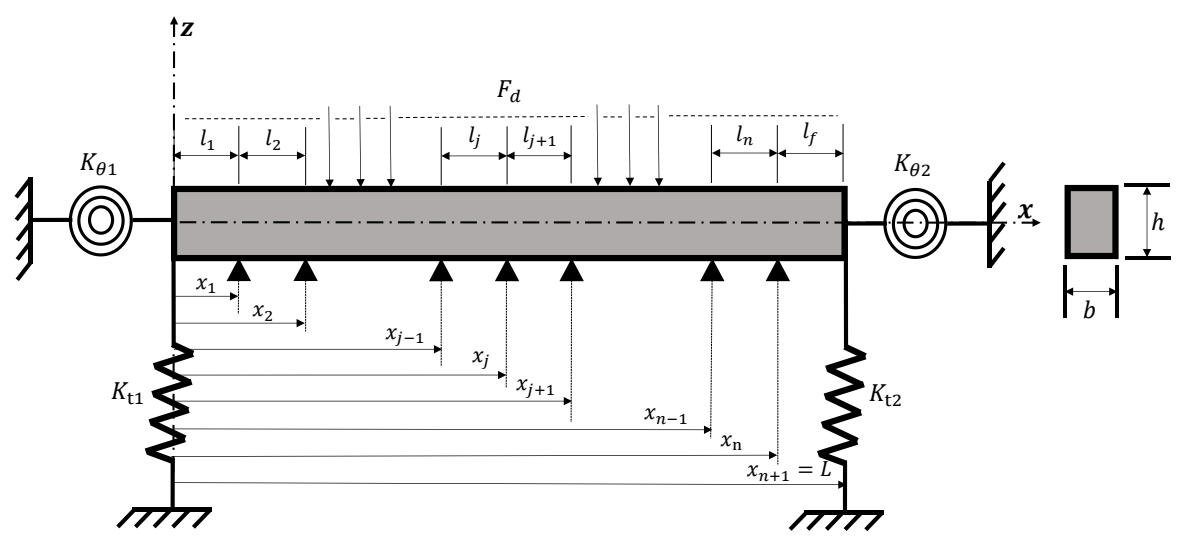

Fig. 1. Physical model of a Multiplan beam with intermediates simple supports and supported by translational and rotational springs at both ends.

Before examining the nonlinear case, we start in this section by determination of the system linear mode shapes, in order to use them as basic functions in the nonlinear theory developed below [1]. The transverse displacement function $\mathbf{w}$ of the beam shown in Fig. 1. can be defined in piecewise by:

$$
\mathbf{w}(\eta)=\left\{\begin{array}{l}
\left.\mathbf{w}_{\mathbf{1}}(\eta) \rightarrow\right] 0, \eta_{1}[ \\
\cdots \\
\left.\mathbf{w}_{\mathbf{j}}(\eta) \rightarrow\right] \eta_{j-1}, \eta_{j}[ \\
\cdots \\
\left.\mathbf{w}_{\mathbf{n}+\mathbf{1}}(\eta) \rightarrow\right] \eta_{n},[[
\end{array}\right.
$$


A closed form solution to this eigenvalue problem can be obtained by employing a transfer matrix method as in [2]. The general solution for transverse vibration in the $(j)^{\text {th }}$ span can be written as:

$$
\begin{gathered}
\mathbf{w}_{j i}(\eta)=\mathbf{a}_{j} \cosh \left(\boldsymbol{\beta}_{i} L\left(\eta-\eta_{j-1}\right)\right)+\mathbf{b}_{j} \sinh \left(\boldsymbol{\beta}_{i} L\left(\eta-\eta_{j-1}\right)\right)+\mathbf{c}_{j} \cos \left(\boldsymbol{\beta}_{\mathrm{i}} L\left(\eta-\eta_{j-1}\right)\right)+\mathbf{d}_{j} \sin \left(\boldsymbol{\beta}_{\mathrm{i}} L\left(\eta-\eta_{j-1}\right)\right) \\
\eta_{j-1} \leq \eta \leq \eta_{j} ; \text { for }: j=1,2, \ldots, \mathrm{n}
\end{gathered}
$$

In which $\quad \beta_{i}=\sqrt[4]{\frac{\rho S \omega_{i}^{2}}{\mathrm{EI}}}$; for $i=1,2, \ldots, \mathrm{N}$ are the eigenvalue parameters for the beam. The constants $\mathbf{a}_{j}, \mathbf{b}_{j}, \mathbf{c}_{j}, \mathbf{d}_{j}$ are determined by the beam end and continuity conditions as follows: The end conditions (at the left end) [3]:

$$
\left.\frac{\mathrm{d}^{3} \mathbf{w}_{\mathbf{1 i}}(\eta)}{\mathrm{d} \eta^{3}}\right|_{\eta 0}=-\left.\mathrm{K}_{\mathbf{t 1}} \mathbf{w}_{\mathbf{1 i}}(\eta)\right|_{\eta 0} ;\left.\frac{\mathrm{d}^{2} \mathbf{w}_{\mathbf{1 i}}(\eta)}{\mathrm{d} \eta^{2}}\right|_{\eta 0}=\left.\mathrm{K}_{\theta 1} \frac{\mathrm{d} \mathbf{w}_{\mathbf{1 i}}(\eta)}{\mathrm{d} \eta}\right|_{\eta 0}
$$

The end conditions (at the right end):

$$
\left.\frac{\mathrm{d}^{3} \mathbf{w}_{(\mathbf{n}+1) \mathbf{i}}(\eta)}{\mathrm{d} \eta^{3}}\right|_{\eta 1}=\left.\mathrm{K}_{\mathrm{t} 2} \mathbf{w}_{(\mathbf{n}+1) \mathbf{i}}(\eta)\right|_{\eta 1} ;\left.\frac{\mathrm{d}^{2} \mathbf{w}_{(\mathbf{n}+1) \mathbf{i}}(\eta)}{\mathrm{d} \eta^{2}}\right|_{\eta 1}=-\left.\mathrm{K}_{\theta 2} \frac{\mathrm{d} \mathbf{w}_{(\mathbf{n}+1) \mathbf{i}}(\eta)}{\mathrm{d} \eta}\right|_{\eta I}
$$

The compatibility conditions are given by [4]:

$$
\begin{array}{r}
\left.\mathbf{w}_{\mathbf{j i}}(\eta)\right|_{\eta j}=\left.\mathbf{w}_{(\mathbf{j}+1) \mathbf{i}}(\eta)\right|_{\eta j}=0 ;\left.\frac{\mathrm{d} \mathbf{w}_{\mathbf{j i}}(\eta)}{\mathrm{d} \eta}\right|_{\eta j}=\left.\frac{\mathrm{d} \mathbf{w}_{(\mathbf{j}+1) \mathbf{i}}(\eta)}{\mathrm{d} \eta}\right|_{\eta j} \\
\left.\frac{\mathrm{d}^{2} \mathbf{w}_{\mathbf{j i}}(\eta)}{\mathrm{d} \eta^{2}}\right|_{\eta \mathbf{j}}=\left.\frac{\mathrm{d}^{2} \mathbf{w}_{(\mathbf{j}+1) \mathbf{i}}(\eta)}{\mathrm{d} \eta^{2}}\right|_{\eta \mathbf{j}} ;\left.\frac{\mathrm{d}^{3} \mathbf{w}_{\mathbf{j i}}(\eta)}{\mathrm{d} \eta^{3}}\right|_{\eta \mathbf{j}}=\left.\frac{\mathrm{d}^{3} \mathbf{w}_{(\mathbf{j}+1) \mathbf{i}}(\eta)}{\mathrm{d} \eta^{3}}\right|_{\eta \mathbf{j}}-\mathrm{R} / \mathrm{EI}
\end{array}
$$

Equations (3-10) and the use of the transfer method used previously in [2] lead to a homogeneous system, whose determinant must be set equal to zero in order to obtain nontrivial solutions defining the natural frequencies. The equation obtained is solved by use of Newton-Raphson method [5].

\subsection{Nonlinear formulation}

The total strain energy $\mathbf{V}$ of the beam can be written as the sum of the axial strain energy due to the nonlinear stretching forces induced by the large deflections $\mathbf{V}_{\mathbf{a}}$, and the strain energy due to bending $\mathbf{V}_{\mathbf{b}}, \mathbf{V}_{\mathbf{a}}, \mathbf{V}_{\mathbf{b}}$ and the kinetic energy $\mathbf{T}$ can be expressed as [6]:

$$
\mathbf{V}_{\mathbf{a}}=\frac{\mathrm{ES}}{8 \mathrm{~L}}\left[\int_{0}^{L}\left(\frac{\partial \mathrm{w}}{\partial x}\right)^{2} \mathrm{~d} x\right]^{2} \mathbf{V}_{\mathbf{b}}=\frac{\mathrm{EI}}{2} \int_{0}^{L}\left(\frac{\partial^{2} \mathrm{w}}{\partial x^{2}}\right)^{2} \mathrm{~d} x \quad \mathbf{T}=\frac{1}{2} \rho \mathrm{S} \int_{0}^{L}\left(\frac{\partial \mathrm{w}}{\partial t}\right)^{2} \mathrm{~d} x
$$

Considering the forced vibration case of a uniform beam. The physical force $\mathbf{F}(x, t)$ excites the modes of the structure via a set of generalized forces $\mathbf{F}_{i}$ which depend on the expression of $\mathbf{F}$, the excitation point for concentrated forces, the excitation length for distributed forces, and the mode considered. 
The generalized forces are given by:

$$
\mathbf{F}_{i}=\int_{\mathrm{S}} \mathbf{F}(x, t) \mathbf{w}_{i}(x) \mathrm{d} x
$$

Consider the beam shown in Fig. $\mathbf{1}$ excited by the distributed harmonic force $\mathbf{F}^{d}$, we have:

$$
\mathbf{F}^{d}(x, t)=\mathbf{F}^{d} \sin (\omega t)
$$

Where $\mathbf{F}_{i}^{d}(t)$ is the corresponding generalized force given by:

$$
\mathbf{F}_{i}^{d}(t)=\mathbf{F}_{0}^{d} \sin (\omega t) \int_{0}^{L} \mathbf{w}_{i}(x) \mathrm{d} x=\mathbf{f}_{i}^{d} \sin (\omega t)
$$

It is well known that the dynamic behaviour of a conservative system may be obtained by applying Hamilton's principle, which, by taking into account the forcing term, may be written as follows [7]:

$$
\delta \int_{0}^{2 \pi / \omega}(\mathbf{V}-\mathbf{T}+\mathbf{F}) \mathrm{d} t=0
$$

Upon assuming harmonic motion and expanding the transverse displacement $w$ in the form of a finite series of basic spatial functions $\left\{\mathbf{w}_{i=1,2, . . n}\right\}$ (the Multi-span beam linear modes determined in the above section) and the time, one obtains:

$$
\mathbf{w}(x, t)=\mathbf{q}(t) \mathbf{w}_{i}(t)=\mathbf{a}_{i} \mathbf{w}_{i} \sin (\omega t)
$$

The following expressions can be written for the potential and kinetic energies (as functionals involving $\left.\mathbf{w}_{i}\right)$, when $\mathbf{w}(x, t)$ is used in the form defined above:

$$
\begin{aligned}
& \mathbf{V}_{\mathbf{a}}=\frac{1}{2} \mathbf{a}_{i} \mathbf{a}_{j} \mathbf{a}_{k} \mathbf{a}_{l} \mathbf{b}_{i j k l} \sin ^{4}(\omega t) ; \mathbf{V}_{\mathbf{b}}=\frac{1}{2} \mathbf{a}_{i} \mathbf{a}_{j} \mathbf{k}_{i j} \sin ^{2}(\omega t) \\
& \mathbf{T}=\frac{1}{2} \omega^{2} \mathbf{a}_{i} \mathbf{a}_{j} \mathbf{m}_{i j} \cos ^{2}(\omega t) ; \mathbf{F}=\mathbf{a}_{i} \mathbf{f}_{i}^{d} \sin ^{2}(\omega t)
\end{aligned}
$$

$\mathbf{b}_{i j k l}$ presents the non-linearity tensor, $\mathbf{k}_{i j}$ denotes the rigidity matrix, and $\mathbf{m}_{i j}$ stands for the mass matrix. The amplitudes $\mathbf{k}_{i j}$ are unknowns as well as the frequency $\omega$.

After calculations, the following non-linear system is obtained:

$$
[\mathbf{K}]\{\mathbf{A}\}+\frac{3}{2}[\mathbf{B}(\{\mathbf{A}\})]\{\mathbf{A}\}-\omega^{2}[\mathbf{M}]\{\mathbf{A}\}=\{\mathbf{F}\}
$$

For obtaining non-dimensional parameters, one puts:

$$
\mathbf{w}(x)=\operatorname{hw}_{i}^{*}\left(\frac{x}{L}\right)=\mathrm{h}_{i}^{*}\left(x^{*}\right) ; \frac{\mathbf{m}_{i j}}{\mathbf{m}_{i j}^{*}}=\rho \operatorname{Sh}^{2} \mathrm{~L} ; \frac{\mathbf{k}_{i j}}{\mathbf{k}_{i j}^{*}}=\frac{\mathrm{EIh}^{2}}{\mathrm{~L}^{3}} ; \frac{\mathbf{b}_{i j k l}}{\mathbf{b}_{i j k l}^{*}}=\frac{\mathrm{EIh}^{2}}{\mathrm{~L}^{3}} ; \frac{\omega^{2}}{\omega^{2 *}}=\frac{\mathrm{EI}}{\rho \mathrm{SL}^{4}}
$$

The dimensionless generalized forces $\mathbf{f}_{i}^{* d}$ is given by:

$$
\mathbf{f}_{i}^{* d}=F^{d} \frac{\mathrm{L}^{4}}{\operatorname{EIh}} \int_{0}^{L} \mathbf{w}_{i}^{*}\left(x^{*}\right) \mathrm{d} x^{*}
$$


Substituting these notations into (23), one obtains the following nonlinear algebraic equation:

$$
\left[\mathbf{K}^{*}\right]\{\mathbf{A}\}+\frac{3}{2}\left[\mathbf{B}^{*}(\{\mathbf{A}\})\right]\{\mathbf{A}\}-\omega^{* 2}\left[\mathbf{M}^{*}\right]\{\mathbf{A}\}=\left\{\mathbf{F}^{*}\right\}
$$

\section{Numerical results and discussion}

In the problem considered herein, we consider a multi-span uniform beam, clamped at both ends, with three intermediate supports equitably distributed, denoted in what follows as C3SS-C.

For clamped ends, we give: $\mathrm{K}_{\mathrm{t} 1}=\mathrm{K}_{\mathrm{t} 2}=\mathrm{K}_{\theta 1}=\mathrm{K}_{\theta 2}=+\infty$

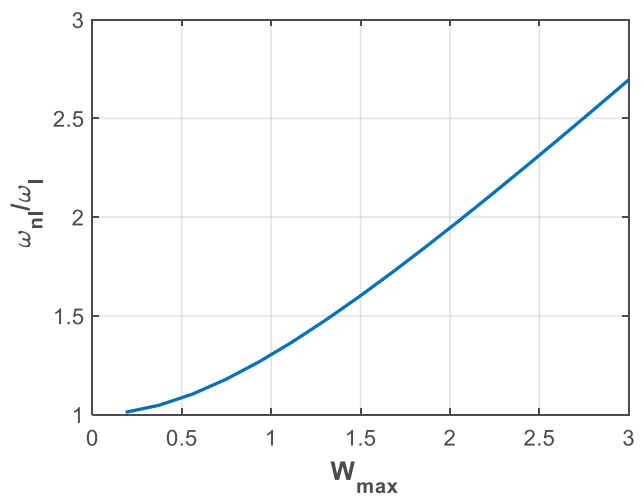

Fig. 2. The first non-dimensional backbone curve of a C-3SS-C beam.

The non-linear frequency ratio versus the non-dimensional vibration amplitude curves of a uniform beam with three intermediate simple supports is shown in figure Fig. 2. for the first non-linear mode shape.
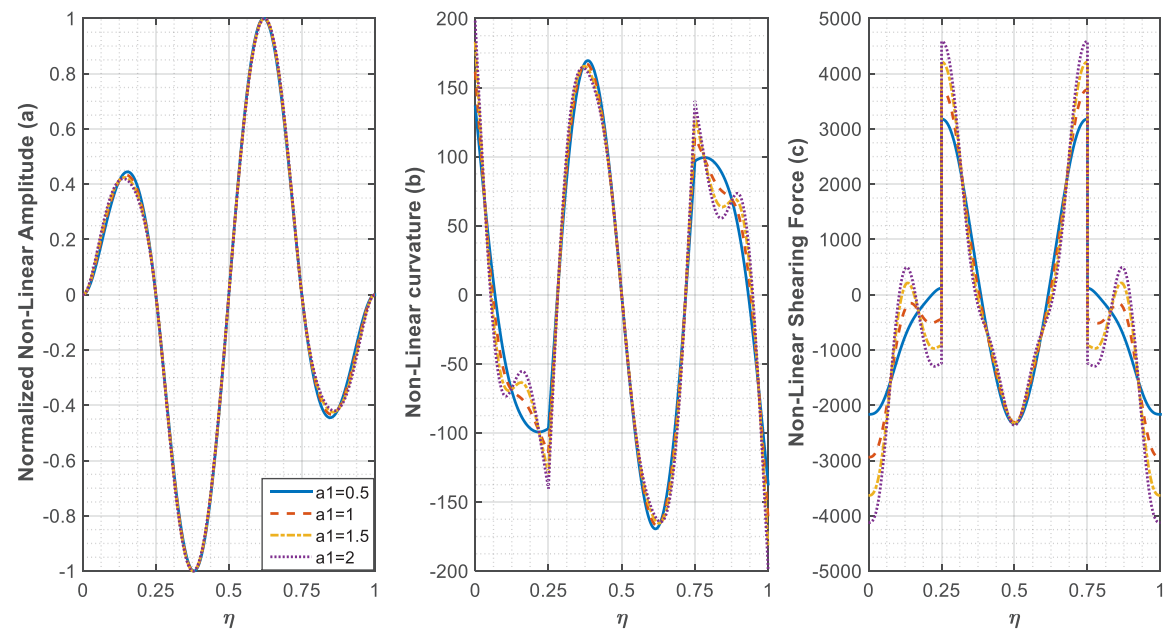

Fig. 3. First Mode, Non-linear curvature and Non-linear shearing force of a C-3SS-C Beam for various vibrations level, in the free vibration case.

The amplitude dependent normalized first nonlinear mode shape, the corresponding curvature and shearing force distributions are plotted Fig. 3. for various vibration amplitudes, the hardening type effect of geometrical non-linearity can been clearly observed. 


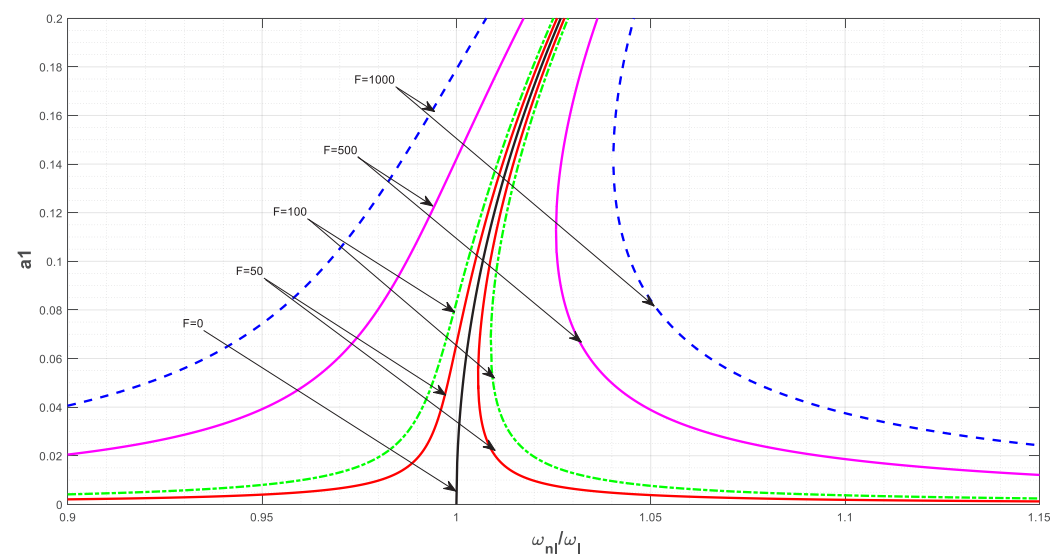

Fig. 4. Nonlinear frequency response functions, based on the single mode approach, of a C-3SS-C beam, for various levels of the harmonic excitation forces.

By assuming a single mode approach used previously in [1], Eq. (30) is presented in Fig. 4 in the neighbourhood of the fourth nonlinear mode shape for various values of the harmonic dimensionless excitation amplitude $f_{1}^{*}$ for the case of a C-3SS-C beam.

\section{Conclusion}

The current work deals with the problem of geometrically nonlinear free and forced vibrations of multi-span beams with intermediate simple supports by use of Hamilton's principle and spectral analysis. First, a study of linear vibrations has been established in order to identify the form of the linear mode shapes, which constitute a basic function in the study of the non-linear case. The intermediate supports effect on the non-linear vibration has been studied and illustrated with by the normalised and the corresponding backbone curves. Using the single mode approach, the effect of the load intensity of the considered beam was examined taking into account the geometrical nonlinearity.

\section{References}

[1] L. Azrar, R. Benamar, and R. G. White, J. Sound Vib., 224, no. 2, pp. 183-207, (1999).

[2] A. Adri, Z. Beidouri, M. El kadiri, and R. Benamar, Asian J. Sci. Technol., 7, no. 02, pp. 2344-2351, (2016).

[3] A. Ahmed and B. Rhali, Int. J. Innov. Res. Sci. Eng. Technol., 6, no. 6, pp. 1103611042, (2017).

[4] Igor A. Karnovskiı̌ and O. I. Lebed, Formulas for structural dynamics: tables, graphs, and solutions. (2001).

[5] J. Kiusalaas, Numerical Methods in Engineering with MATLAB, Second Edi. (2010).

[6] M. El Kadiri, R. Benamar, and R. G. White, J. Sound Vib., 249, no. 2, pp. 263-305, (2002).

[7] M. Géradin and D. J. Rixen, Mechanical Vibrations: Theory and Application to Structural Dynamics. (2015). 